Research Article

\title{
Effects of Porosity on the Static Compression of Foam Buffer Materials of Plant Fiber and Their Numerical Model
}

\author{
Chongqian Huang $\mathbb{D}$, Xueke Zhang $\mathbb{D}$, and Zhengqi Liu \\ College of Mechanical and Electrical Engineering, Guangxi Science \& Technology Normal University, Laibin 546199, China \\ Correspondence should be addressed to Chongqian Huang; huangcq0772@163.com
}

Received 14 January 2021; Accepted 29 July 2021; Published 9 August 2021

Academic Editor: Xin Xue

Copyright ( 92021 Chongqian Huang et al. This is an open access article distributed under the Creative Commons Attribution License, which permits unrestricted use, distribution, and reproduction in any medium, provided the original work is properly cited.

\begin{abstract}
In recent years, researchers have investigated plant fiber foam buffer package materials for their environmental advantages, which include their ability to completely biodegrade and their rich raw materials. The porosity of buffer materials is an important factor that affects a plant's static compression property. In this study, foam buffer package materials of plant fiber were developed using bleached bagasse pulp as the main raw material. A digital microscope was used to observe the porosity structures of the foam buffer materials, and the porosity was calculated via binary image processing using Matlab software. The effects of porosity on the static compression properties of cushion materials were discussed, and a numerical model was established. The results indicated that the threshold value chosen affected the calculation of the porosities of buffer materials, which in turn have significant effects on their specific static compression properties. The variation trend of the stress-strain curve fits the hyperbolic tangent function, according to which a numerical model was established. The results of this experiment were in accordance with the theoretical values, which will support the theoretical design of foam buffer package materials of plant fiber.
\end{abstract}

\section{Introduction}

Plastics have caused increasingly serious pollution problems, thereby making it necessary to develop a degradable natural buffer material to replace the current widely used, nondegradable, plastic foaming material used in packaging and other applications $[1,2]$. Currently, commonly used environmentally friendly degradable buffer materials include molded pulp and honeycomb paperboard products. Since these products involve the papermaking process in their production, the cushioning cavity structure can improve its cushioning properties. However, these buffer materials have the disadvantages of poor elasticity, elastic modulus, cushion coefficients, greater friability, and specific weights. In addition, their production costs are much higher than those of plastic foam products, which also restricts their distribution and use.

However, foam cushioning package materials made from plant fibers (e.g., agricultural waste, wastepaper, cardboard, and other plant fiber materials) and starch additives are a new type of green packaging material [3]. Its advantages include simple manufacturing technology, low cost, wide material sources, and better shock isolation performance than molded pulp products [4]. It can not only be made into lined shockproof packaging material but can also replace expanded polystyrene (EPS) filling particles, as its performance is similar to that of EPS products. The new buffer material is a more sustainable and environmentally friendly product, which effectively addresses issues arising from the scarcity of resources and the contradiction between environmental pollution and improvements in quality of life because it undergoes natural degradation completely. In recent years, besides increasing concerns about environmental pollution, natural buffer materials found in plant fibers have been the focus of greater research and development efforts, especially in the field of preparation technologies [5-7].

Generally, buffer package materials should demonstrate impact energy absorbability, vibration absorbability, good resilience, compression creep resistance, mildew resistance, 
oil resistance, antistatic properties, wear resistance, flexibility, and acid and alkali resistance [8-14]. Foam buffer materials made from plant fibers contain a large number of porous structures, which easily adsorb moisture from the air, thus potentially changing the mass and volume of the structure under natural environmental conditions [15]. Therefore, the results under different humidity conditions might have a larger error, so the apparent density cannot reflect the real properties of the foam buffer materials [16]. In porous materials, the porosity is an important structural parameter that reflects the pore density of the buffer materials without the influence of environmental factors. Several methods can be used to measure the porosity of buffer materials: the microscope scanning grid method, mercury intrusion method [17], nitrogen adsorption, liquid infiltration methods, and image analysis methods.

The static compression properties of foam buffer materials have a great impact on the strength of bonding between the fibers of the materials as well as its porosity [18]. Generally, buffer materials with a larger rate of porosity have the static compression characteristic curve closer to the $X$ axis. In this study, foam buffer packaging materials were developed from plant fiber using bleached bagasse pulp as the main raw material. A digital microscope was used to observe the porosity structure of the cushion materials, and the porosity was calculated via binary image processing using Matlab software. The effects of the porosities of the cushion materials were discussed and the model parameters were identified using experimental data to establish the static compression characteristic equations.

\section{Experimental Methods}

2.1. Materials. The materials included bleached bagasse pulp from Guangxi Nanning Sugar Industry Co., Ltd.; cationic starch (99\%, CS-8) from Guangxi Mingyang Biochemical Technology Co., Ltd.; sodium bicarbonate, AR, from the Chengdu Kelong Chemical Reagent Factory; polyvinyl alcohol, 1788, from the Chengdu Kelong Chemical Reagent Factory; and industrial grade magnesium stearate (AR) from Sinopharm Chemical Reagent Co., Ltd.

2.2. Equipment. The equipment included the INSTRON 8801 material testing system from Instron (Shanghai) Test Equipment Trading Co., Ltd. and the DMLS2 microscopy and image analysis system from German Leica Instruments, Inc.

2.3. Preparation of Foam Buffer Materials. Bleached bagasse pulp with a beating degree of $73^{\circ} \mathrm{SR}$ was used as the main raw material. (The length and content of the number-average fine fiber, $0.40 \mathrm{~mm}, 37.98 \%$. The length and content of the weight-average fine fiber, $1.46 \mathrm{~mm}, 10.79 \%$.) The cationic starch and polyvinyl alcohol aqueous solution were stirred at $90^{\circ} \mathrm{C}$ for $30 \mathrm{~min}$ separately and then mixed to form a paste. Then, a certain amount of the dispersed fiber pulp and magnesium stearate $(1.50 \%$ of the dry weight of material) was added to the paste and stirred until consistency. After the mixed solution was cooled to room temperature, sodium bicarbonate was added as a foaming agent. After stirring and mixing to achieve a uniform consistency, the mixture was moved into the mold [19]. Additionally, the mass ratio of fiber, cationic starch, and polyvinyl alcohol was approximately $7: 2: 1$. The mixture was then foamed into buffer materials with different porosities in the microwave oven, based on the different foaming agents used and various microwave foaming times.

After being molded, the buffer materials were removed from the mold and placed in an oven to dry fully. The preparation process is shown in Figure 1.

2.4. Microscopic Image Acquisition and Analysis. The material was cross-sectionally cut with a sharp knife edge to obtain a sheet-like observation sample whose microstructure was observed using a digital microscope to obtain the microstructure image of the corresponding sample. The acquired images were processed using Matlab software. The microstructure image is gray-scaled, and an appropriate threshold is selected to binarize the image. The Matlab software was used to calculate the number of white pixels in the image, which in turn helped calculate the percentage of white pixels compared to the total pixels to obtain the corresponding image porosity.

Multiple points were randomly selected for each sample and their cross-sectional porosity was calculated. Finally, the average value of the multiple points was calculated to obtain the porosity of the sample.

2.5. Measurement of Static Compression Property. The static compression property was measured according to the Chinese national standards GB 8168-2008, Method A, the static compression test method used for buffer package materials. In the experiment, the load applied to the samples was gradually increased at a velocity of $(12 \pm 3) \mathrm{mm} / \mathrm{min}$ along the direction of thickness; the compression force and the corresponding deformation during the sample compression process were recorded. Thus, the stress-deformation curve can be obtained. To eliminate the influence of the material size, the stress-deformation curve was converted into the stress-strain curve according to the following equations:

$$
\sigma=G(\rho) f(\varepsilon),
$$

where $\sigma$ is the compression force (Pa), $P$ is the compression load $(\mathrm{N})$, and $A$ is the loading area $\left(\mathrm{mm}^{2}\right)$.

$$
\sigma=a_{1} \operatorname{th}\left(a_{2} \varepsilon\right)+a_{3} \tan \left(a_{4} \varepsilon\right),
$$

where $\varepsilon$ is the compressive strain (\%), $T$ is the thickness of the samples before compression $(\mathrm{mm})$, and $T_{i}$ is the thickness of the samples after compression $(\mathrm{mm})$.

\section{Results and Discussion}

3.1. Measurements of Porosity. While calculating the porosities of the structures of the buffer materials, when a given 


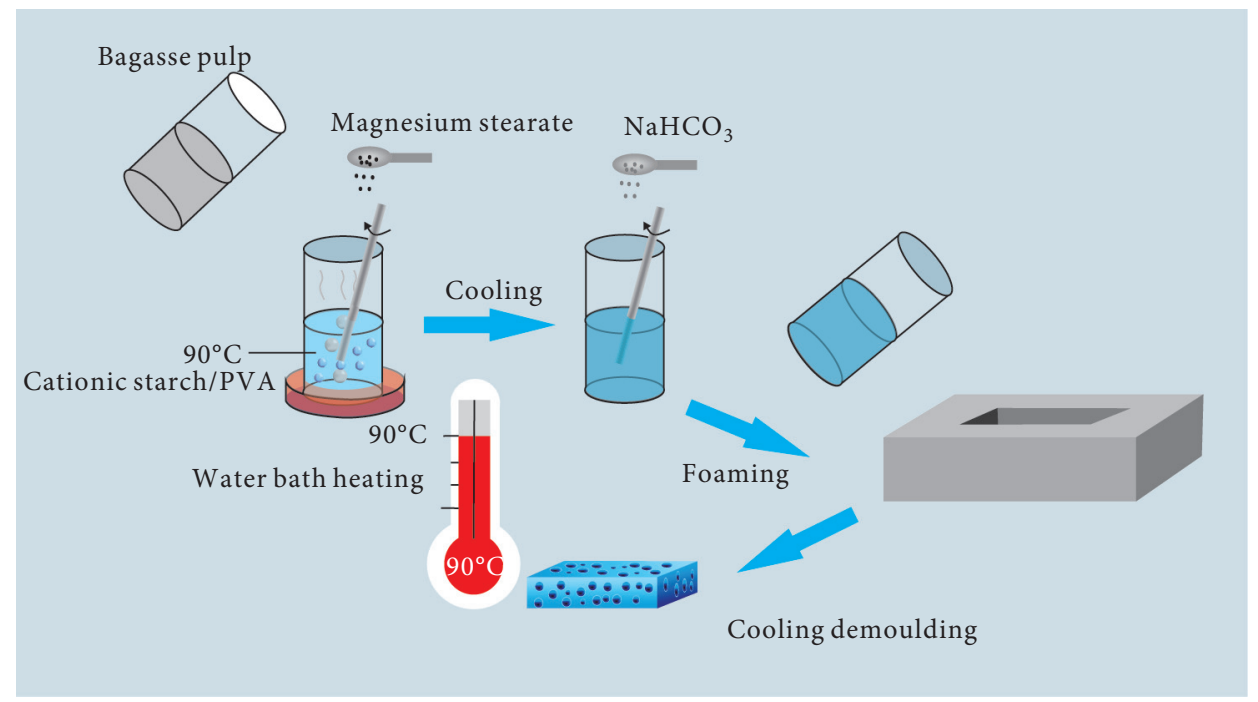

FIgURE 1: Flow chart of the preparation of foam buffer.

binarization threshold was chosen, it could be observed in the range of the black pixels that the porosities of the binarization image could be zoomed in or out from the original images [20], leading to larger errors in the final porosity measurements. In practical calculations, multiple values should be chosen to ensure that the selected image is closest to the original image after binarization, and the most appropriate threshold to calculate the porosity of the buffer materials should be selected.

The image results are shown in Figure 2. The image with a threshold of 0.4 (Figure 2(c)) and the ash processing image in Figure 2(a) have the same pore distribution, but the image with a threshold of 0.3 (Figure 2(b)) incorrectly identifies some fiber structures. For the porosity, the image (Figure 2(d)) with a processing threshold of 0.5 cannot distinguish some microporous structures in the sample. Therefore, image $c$ is selected for calculating the porosity, which is $49.4 \%$.

In addition, there are a significant number of open-pore structures inside the materials, and a large number of fibers are entangled between them. This is mainly due to a large amount of cationic starch adsorbed on the surface of the fiber, making it easier for the fibers to come in contact with each other [21,22]. The presence of cationic starch causes the fibers to bind more closely, forming a layered fiber microstructure and enhancing the stability of the fiber network structure. Alternatively, it may also be because polyvinyl alcohol and cationic starch both play the role of binding fibers.

3.2. Porosities of the Different Samples. Foam buffer samples with different porosities were prepared using various foaming agents and microwave foaming times. Appropriate binarization thresholds were chosen to calculate the porosities using Matlab software. For each sample, 8-10 different cross-sections were measured, and the average value of the porosities was calculated, with the results shown in Figure 3.
As shown in Figure 3, the internal pore structure of the cushioning material varies with the amount of foaming agent and microwave foaming time. As the amount of foaming agent and microwave foaming time increases, the porosity of the cushioning material gradually increases. This may be due to the diffusion of gas during the microwave foaming process and the increase in air pressure in the pores, which causes the fibers to squeeze together and bond [23]. Therefore, the longer the diffusion time and the greater the increase in the amount of gas generated during the foaming process, the larger is the fiber spacing. This also increases the porosity. The amount of foaming agent has a significant influence on the porosity of the material. However, the increase in foaming time had a smaller change in porosity compared to the amount of foaming agent. It can be proved that the influence of the foaming agent on the porosity of the material is far greater than the foaming time [15].

\subsection{Static Compression Property Analysis of Different} Porosities. With reference to the porosity of the material, the static compression characteristic curves of samples with different porosities are compared, and the stress-strain curves of the samples with the same porosity are selected for average processing. A total of five groups of buffer materials with different porosities were obtained. The results are shown in Table 1.

According to the test of the compressive performance of average porosity (Table 1), the average porosity of samples S1-S5 was $64.8 \%, 69.7 \%, 75.4 \%, 80.1 \%$, and $84.5 \%$, respectively.

The static compression properties of the different porosities at $64.8 \%, 69.7 \%, 75.4 \%, 80.1 \%$, and $84.5 \%$ from samples $S 1$ to $S 5$ were tested in this study, and the static compression characteristic curves were drawn. The different stress-strain curves are displayed in Figure 4.

As shown in Figure 4, the static compressive stress-strain curve of the foam buffer material found in plant fiber can be divided into three stages: the range between 0 and $10 \%$ of the 


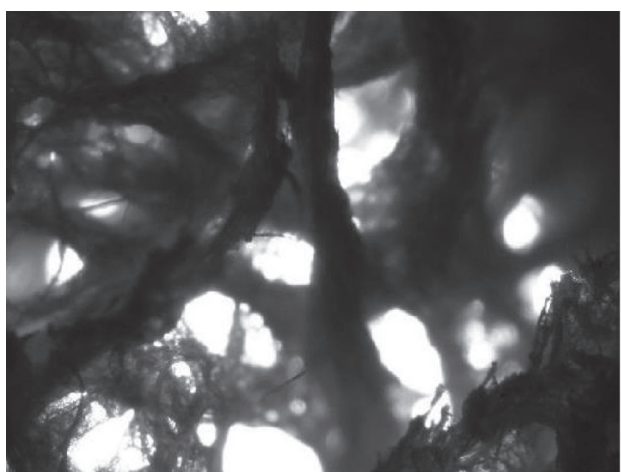

(a)

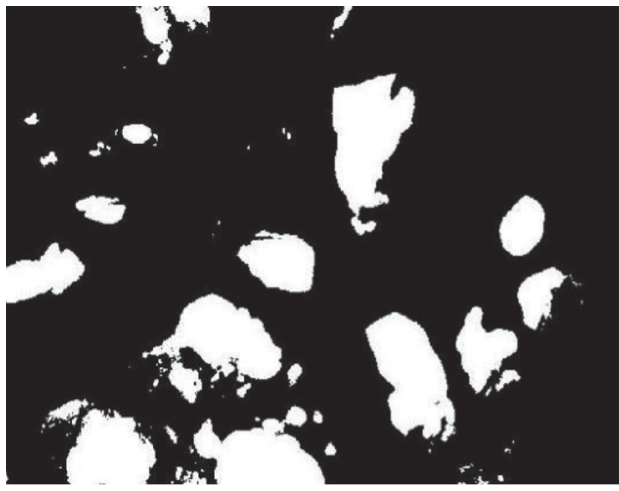

(c)

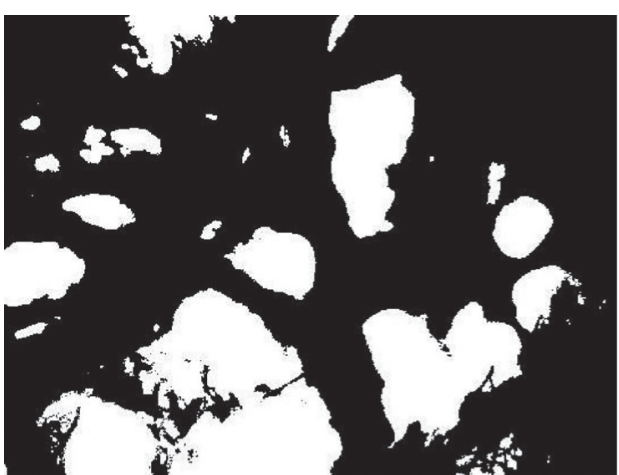

(b)

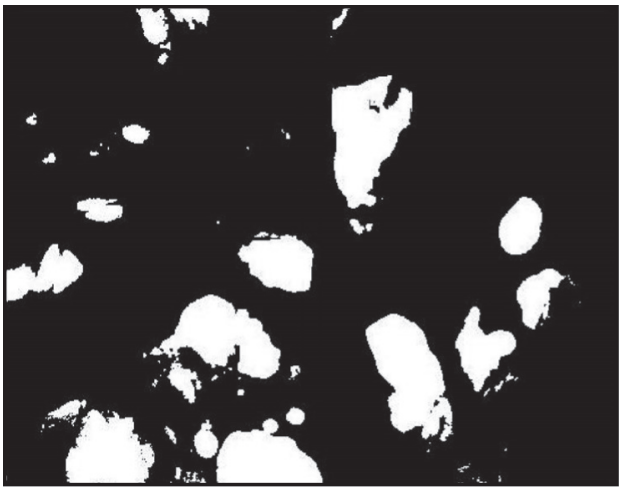

(d)

FIgURE 2: Real images and binary images with different thresholds. (a) Image of ashing processing. The image of the sample at the threshold values of (b) 0.3 , (c) 0.4 , and (d) 0.5 .

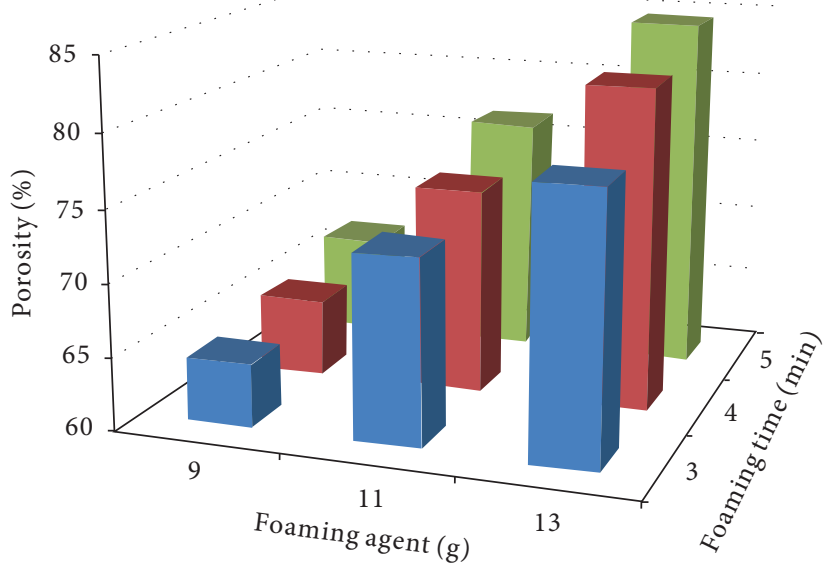

FIGURE 3: Effects of different foaming agents and microwave foaming time on the porosity of buffer materials.

strain is the smallest strain elastic deformation zone; that is, the stress-strain curve is basically linear; the range between 10 and $50 \%$ of the strain is known as the platform area (or yield) where the stress force change is not large when the strain changes sharply, and the curves are relatively flat. After $50 \%$, the curve is tangent; when the strain increases, the stress forces also increase sharply and tend to infinity, which is known as the compaction area (or density). This may be because the foamed material has a certain degree of elasticity, and when the deformation is small, it falls within the deformation range of the foamed material. As the deformation increases, the polymer bond of the material itself generates a huge force [24].

In addition, when the material is under the same strain conditions, the smaller the porosity, the greater the stress; that is, the tighter the fiber cross-links, the greater the corresponding stress in the material $[25,26]$. Therefore, the form buffer materials of plant fiber can absorb more kinetic 
TABle 1: Data of void fraction of different samples.

\begin{tabular}{lc}
\hline Sample & Average porosity (\%) \\
\hline S1 & 64.8 \\
S2 & 69.7 \\
S3 & 75.4 \\
S4 & 80.1 \\
S5 & 84.5 \\
\hline
\end{tabular}

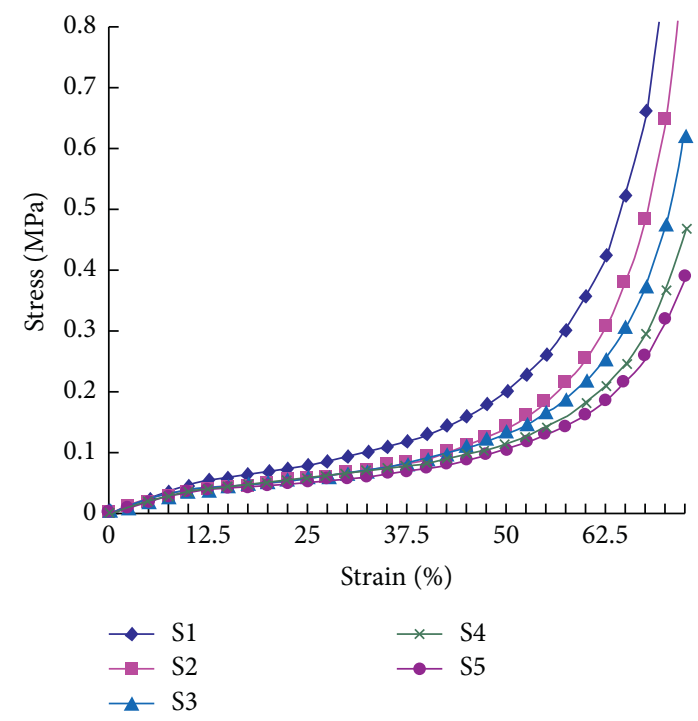

FIGURE 4: Stress-strain curves of cushion materials of different porosities.

energy from the outside. In addition, the flag stage of the low-porosity buffer materials was narrow and the corresponding stress force was higher because the buffer materials with lower porosity rates compacted more easily, resulting in a shorter yielding phase.

\subsection{Establishment of the Static Compression Model of Buffer} Materials. This study established a static compression model because, to meet practical need, the application of plant fiber-shaped cushioning materials has been widely promoted. Based on the classical mechanics model of the buffer material of plastics, a static compression model equation of the buffer materials of plant fiber was assumed and the parameters of the model were estimated using experimental data from static compression test data fitting. The characteristic equation was then established, which describes the static compression property of foam buffer materials found in plant fibers more effectively.

The existing static compression model of buffer materials mostly belongs to the semiempirical formula. Sherwood and Frost [27] put forward the model of an expression formula that takes environmental temperature and density into consideration, which is shown in the following equation:

$$
\sigma=H(T) G(\rho) f(\varepsilon) .
$$

Here, $H(T)$ and $G(\rho)$ indicate that the environment temperature $T$ and density $\rho$ have an impact on stress, and their impact is single, and $f(\varepsilon)$ is the shape function, which simulates the material deformation process. Due to the high porosity of the material at room temperature, the temperature change generated during the compression process has very little effect on the stress of the material, so the effect of temperature is ignored. So formula (3) can be simplified as follows:

$$
\sigma=G(\rho) f(\varepsilon) .
$$

From equation (4), we can see that the static compression test of buffer materials of different pore densities is a function of strain. Referring to the classical mechanics model of the buffer materials and considering the changing trend of the experimental stress-strain curve, static compression can be understood to use a hyperbolic tangent. The tangent function can be used to fit the static compression curve equation of the plant fiber foam buffer materials. A model of the static compression characteristic equation was established in the following equation:

$$
\sigma=a_{1} \operatorname{th}\left(a_{2} \varepsilon\right)+a_{3} \tan \left(a_{4} \varepsilon\right) .
$$

In equation (5), a hyperbolic tangent function was used to reflect the relatively flat part of the stress-strain curve, where $a_{1}$ and $a_{2}$ are only related to the elastic constant of the materials. The tangent function is used to reflect the dramatic change in strain in the stress-strain curve; among them, $a_{3}$ and $a_{4}$ are only related to the elastic constants of the materials.

3.5. Fitting Recognition of the Static Compression Model of Buffer Materials. A nonlinear least square method was used for curve fitting, and the lsqcurvefit function in Matlab software was used in the fitting recognition process. According to the stress and strain experiment of the samples with a porosity of $84.5 \%$, the results for the numerical recognition characteristic equation parameters are $a_{1}=0.0159, a_{2}=18.7668, a_{3}=0.0598$, and $a_{4}=1.9614$ and the residual sum of the squares is 0.00022 . In other words, the static compression model equation of the samples is $\sigma=0.0159 \operatorname{th}(18.7668 \varepsilon)+0.0598 \tan (1.9614 \varepsilon)$. This equation is used to calculate the different strain conditions of stress and draw the stress-strain curve of the buffer materials, as shown in Figure 5. Here, a smooth curve was formed according to the model calculation value and the scatter line was formed according to the experimentally measured values.

As shown in Figure 5, under the model equations, the calculated stress values under different strain conditions are basically the same as those obtained in the experiment, without much difference. In addition, the sum of the squared residuals of the equation in the figure is very small.

In other words, the dispersion between the measured value and the fitted curve is very low, indicating that the static compression characteristic equation in the figure has a high accuracy rate. Therefore, the fitting equation has a high reference value for the compression performance of sample S1. 


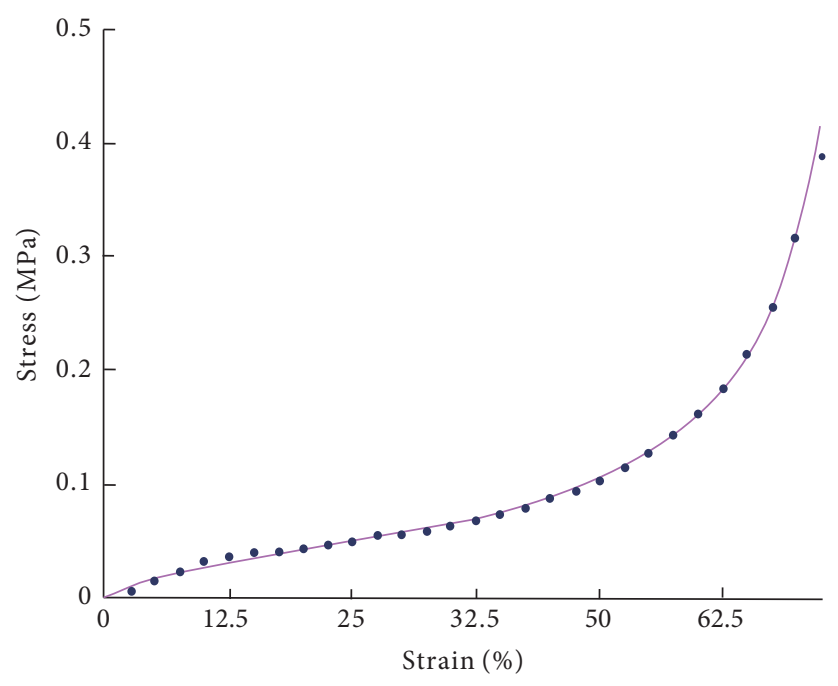

- Experimental value

_ Calculated value

Figure 5: Comparison of the stress-strain curves of the experimental values and calculated value.

TABLE 2: Static compression characteristic equation of different porosity samples.

\begin{tabular}{lccc}
\hline Sample & The average porosity $(\%)$ & The characteristic equation & Residual sum of squares \\
\hline S1 & 64.8 & $\sigma=0.0039 \operatorname{th}(20.8588 \varepsilon)+0.1299 \tan (2.0281 \varepsilon)$ & 0.0034 \\
S2 & 69.7 & $\sigma=0.002 \operatorname{th}(17.3505 \varepsilon)+0.0896 \tan (2.0455 \varepsilon)$ & 0.0016 \\
S3 & 75.4 & $\sigma=0.0112 \operatorname{th}(18.4911 \varepsilon)+0.0785 \tan (2.0038 \varepsilon)$ & 0.00051 \\
S4 & 80.1 & $\sigma=0.0206 \operatorname{th}(14.4173 \varepsilon)+0.0631 \tan (1.9868 \varepsilon)$ & 0.00022 \\
S5 & 84.5 & $\sigma=0.0159 \operatorname{th}(18.7668 \varepsilon)+0.0598 \tan (1.9614 \varepsilon)$ & 0.00022 \\
\hline
\end{tabular}

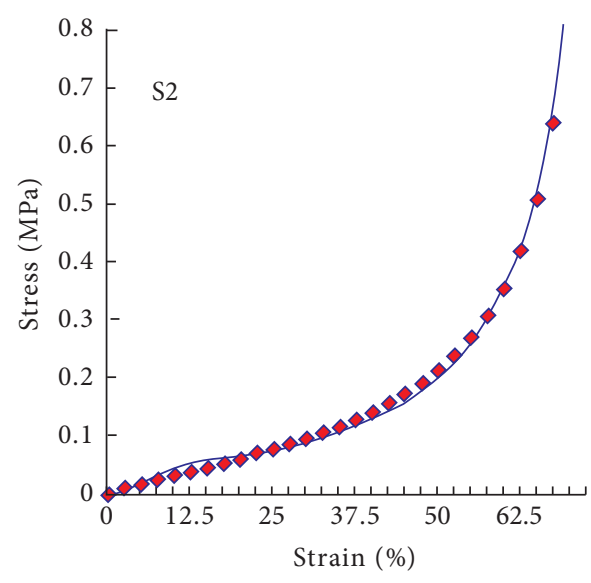

_ Experimental values

$\checkmark$ Calculated values

(a)

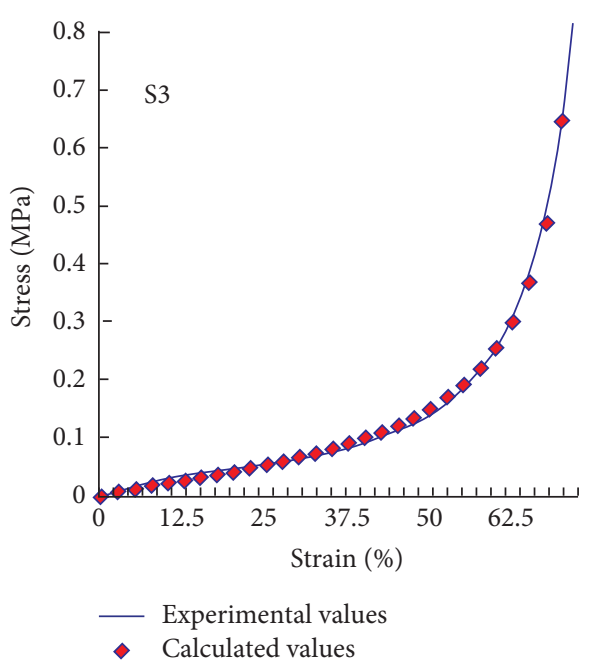

(b)

FIgURE 6: Continued. 


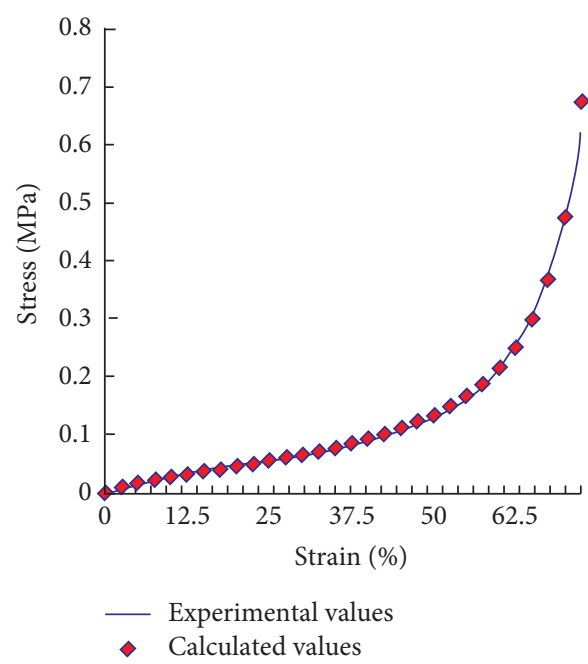

(c)

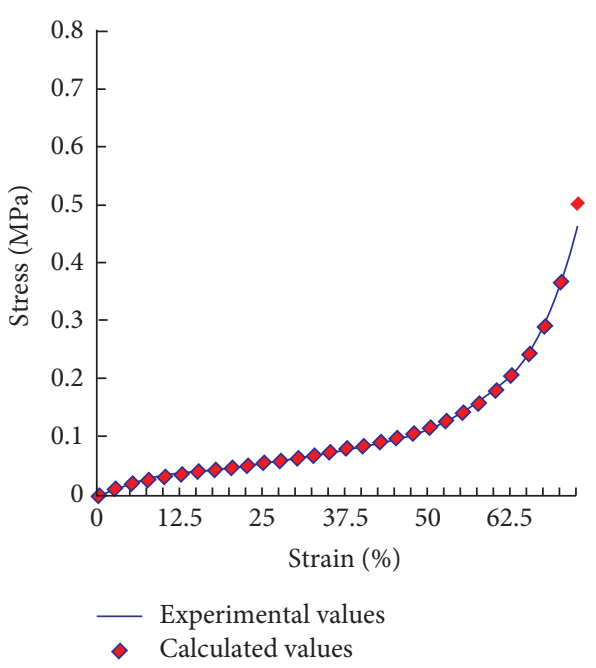

(d)

Figure 6: Comparison of the stress-strain curves of the different samples of the experimental values and calculated value.

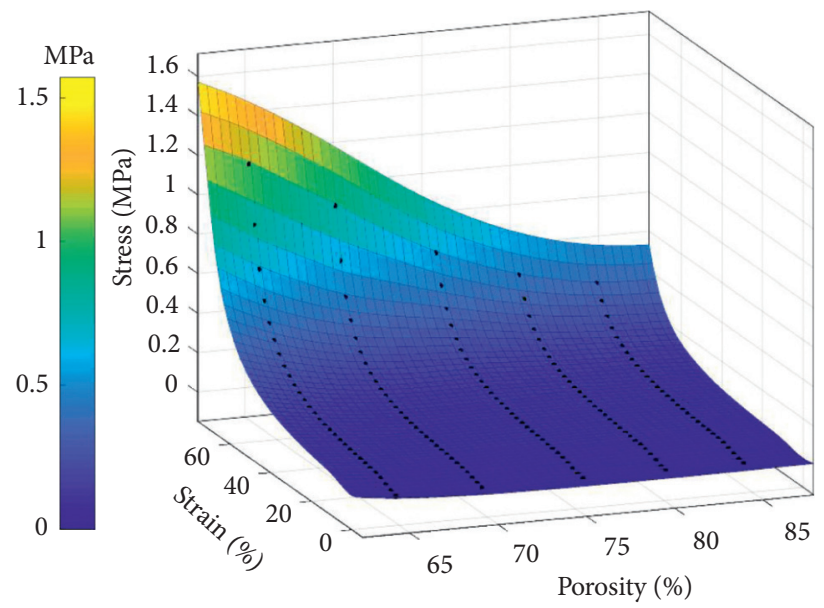

FIgURE 7: Surface fitting of stress-strain curves and porosities.

Using the same method, Table 2 demonstrates the characteristic equation of the static compression characteristic equation of the samples with different porosities.

The stress-strain curve of the static compression of the experimental value was calculated using the same method shown in Figure 6.

For samples with different porosities $(S 2, S 3, S 4, S 5)$, curves were drawn according to the model characteristic equations obtained by least squares fitting, as shown in Figure 6. Similar to the fitting equation of $S 1$, the fitting equations of $S 2-S 5$ have a higher degree of coincidence in the curves, which is of reference value in the study of stressstrain of materials, even if their maximum pressures are different. Therefore, the fitting equation has reference significance for the compressive stress-strain changes of foamed materials. The results show that the characteristic equation is reasonably accurate and provides a basis for analyzing the cushioning performance of cushioning materials.

The surface fitting of stress-strain curves and porosities was fitted by interpolation method using the $c f$ tool of Matlab software. The results are shown in Figure 7. In practice, interpolation methods can be used according to these surfaces to predict the stress-strain relationships of certain porosity materials of plant fiber.

\section{Conclusions}

(1) When image processing technology is used to analyze the porosity of the foam buffer materials, the chosen binarization threshold can affect the calculation of the porosity. In the application of the aforementioned calculations, multiple sample values should be chosen to ensure that the image used is 
closest to the original image after binarization, and the most appropriate threshold should be selected to calculate the porosity of the buffer materials.

(2) The internal pore structure of the buffer materials changed with different dosages of foaming agents and various microwave foaming times. The greater the dosage of the foaming agent and the longer the microwave foaming time, the greater the porosity of the buffer materials.

(3) The static compressive stress-strain curve of the foam buffer materials found in plant fibers can be divided into three stages, which include the small strain elastic deformation zone, the platform area, and the compaction area.

(4) Based on the above experiments, the static compression features of foam buffer materials with different porosities were analyzed and the static compression characteristic equation was established. Moreover, the equation parameter was identified using experimental data, and finally, the rationality of the mode was proven. The static compression model equation is $\sigma=a_{1} \operatorname{th}\left(a_{2} \varepsilon\right)+a_{3} \tan \left(a_{4} \varepsilon\right)$.

\section{Data Availability}

The original data used to support the findings of this study have not been made available because they are part of an ongoing study.

\section{Conflicts of Interest}

The authors declare that they have no conflicts of interest.

\section{Acknowledgments}

This work was supported by funding from the Scientific Research and Technology Development Project of Laibin City (grant number 20LKZ202409), Guangxi Science \& Technology Normal University (grant number 2020GKSYGZ01), and Guangxi Education Department (grant number 2021JGZ169).

\section{References}

[1] H. Kargarzadeh, J. Huang, N. Lin et al., "Recent developments in nanocellulose-based biodegradable polymers, thermoplastic polymers, and porous nanocomposites," Progress in Polymer Science, vol. 87, pp. 197-227, 2018.

[2] B. Jeon, H. K. Kim, S. W. Cha, S. J. Lee, M. S. Han, and K. S. Lee, "Microcellular foam processing of biodegradable polymers-review," International Journal of Precision Engineering and Manufacturing, vol. 14, no. 4, pp. 679-690, 2013.

[3] N. Soykeabkaew, C. Thanomsilp, and O. Suwantong, "A review: starch-based composite foams," Composites Part A: Applied Science and Manufacturing, vol. 78, pp. 246-263, 2015.

[4] J. Li, X. Yang, H. Xiu et al., "Structure and performance control of plant fiber based foam material by fibrillation via refining treatment," Industrial Crops and Products, vol. 128, pp. 186-193, 2019.
[5] Y. Chen, K. Zhang, T. Zhang et al., "Effect of softening treatments on the properties of high-density cylindrical luffa as potential mattress cushioning material," Cellulose, vol. 26, no. 18, pp. 9831-9852, 2019.

[6] J. Li, X. Yang, H. Xiu et al., "Structure and performance control of plant fiber based foam material by fibrillation via refining treatment," Industrial Crops and Products, vol. 128, pp. 186-193, 2019.

[7] R. A. Ilyas, S. M. Sapuan, M. L. Sanyang, M. R. Zainudin, and E. S. Zainudin, "Nanocrystalline cellulose as reinforcement for polymeric matrix nanocomposites and its potential applications: a review," Current Analytical Chemistry, vol. 14, no. 3, pp. 203-225, 2018.

[8] G. M. Glenn, S. H. Imam, W. J. Orts, and A. K. Klamczynski, "Fiber reinforced starch foams," Society of Plastics Engineers, vol. 2, pp. 2484-2488, 2004.

[9] N. Soykeabkaew, P. Supaphol, and R. Rujiravanit, "Preparation and characterization of jute- and flax-reinforced starch-based composite foams," Carbohydrate Polymers, vol. 58, no. 1, pp. 53-63, 2004.

[10] X. Q. Dong, D. J. Sun, G. P. Liu, C. B. Cao, and X. R. Jiang, "Aqueous foam stabilized by hydrophobically modified cellulose and alkyl polyoxyethyl sulfate complex in the presence and absence of electrolytes," Colloids and Surfaces A: Physicochemical and Engineering Aspects, vol. 345, no. 1-3, pp. 58-64, 2009.

[11] C. Ge, "Theory and practice of cushion curve: a supplementary discussion," Packaging Technology and Science, vol. 32, no. 4, pp. 185-197, 2019.

[12] Y. Q. Su, B. Yang, J. G. Liu et al., "Prospects for replacement of some plastics in packaging with lignocellulose materials: a brief review," Bioresources, vol. 13, no. 2, pp. 4550-4576, 2018.

[13] I. Singh, S. K. Samal, S. Mohanty, and S. K. Nayak, "Recent advancement in plant oil derived polyol-based polyurethane foam for future perspective: a review," European Journal of Lipid Science and Technology, vol. 122, no. 3, Article ID 1900225, 2020.

[14] M. Nofar and C. B. Park, "Poly (lactic acid) foaming," Progress in Polymer Science, vol. 39, no. 10, pp. 1721-1741, 2014.

[15] C. X. Huang, Q. Zhu, C. C. Li, W. Lin, and D. J. Xue, "Effects of micronized fibers on the cushion properties of foam buffer package materials," Bioresources, vol. 9, no. 4, pp. 5940-5950, 2014.

[16] Y. Y. Luo, S. L. Xiao, and S. L. Li, "Effect of initial water content on foaming quality and mechanical properties of plant fiber porous cushioning materials," Bioresources, vol. 12, no. 2, pp. 4259-4269, 2017.

[17] M. J. Moura, P. J. Ferreira, and M. M. Figueiredo, "Mercury intrusion porosimetry in pulp and paper technology," Powder Technology, vol. 160, no. 2, pp. 61-66, 2015.

[18] P. Liu, F. Li, J. Li, K. Guan, G. Li, and G. Anfu, "Effect of starch plasticizing/fiber processing on the mechanical properties of biomass cushion packaging composites," Journal of Biobased Materials and Bioenergy, vol. 8, no. 2, pp. 214-220, 2014.

[19] R. L. Shogren, J. W. Lawton, and K. F. Tiefenbacher, "Baked starch foams: starch modifications and additives improve process parameters, structure and properties," Industrial Crops and Products, vol. 16, no. 1, pp. 69-79, 2002.

[20] J. H. Liu, X. Q. Wu, and Q. W. Guo, "Comparison of porosity detection method of the composites," Fiber Composites, vol. 26, no. 1, pp. 29-31, 2009.

[21] A. Nechwatal, C. Michels, B. Kosan, and M. Nicolai, "Lyocell blend fibers with cationic starch: potential and properties," Cellulose, vol. 11, no. 2, pp. 265-272, 2004. 
[22] E. Ferstl, M. Gabriel, F. Gomernik et al., "Investigation of the adsorption behavior of jet-cooked cationic starches on pulp fibers," Polymers, vol. 12, no. 10, p. 2249, 2020.

[23] K. Heinze, X. Frank, V. Lullien-Pellerin, M. George, F. Radjai, and J. -Y. Delenne, "Stress transmission in cemented bidisperse granular materials," Physical Review, vol. 101, no. 5, Article ID 052901, 2020.

[24] S. He, C. Liu, X. Chi et al., "Bio-inspired lightweight pulp foams with improved mechanical property and flame retardancy via borate cross-linking," Chemical Engineering Journal, vol. 371, pp. 34-42, 2019.

[25] C. Durir, M. Wynn, M. Balestrat et al., "Open-celled silicon carbide foams with high porosity from boron-modified polycarbosilanes," Journal of the European Ceramic Society, vol. 39, no. 16, pp. 5114-5122, 2019.

[26] G. Sun, E. Wang, J. Zhang, S. Li, Y. Zhang, and Q. Lib, "Experimental study on the dynamic responses of foam sandwich panels with different face sheets and core gradients subjected to blast impulse," International Journal of Impact Engineering, vol. 135, Article ID 103327, 2020.

[27] J. A. Sherwood and C. C. Frost, "Constitutive modeling and simulation of energy absorbing polyurethane foam under impact loading," Polymer Engineering \& Science, vol. 32, no. 16, pp. 1138-1146, 1992. 